\title{
Serum angiostatin and endostatin levels in patients with granulomatosis with polyangiitis and immune complex small vessel vasculitis
}

\author{
Anna Kotulska-Kucharz, Magdalena Kopeć-Mędrek, Eugeniusz J. Kucharz \\ Department of Internal Medicine, Rheumatology and Clinical Immunology, Medical University of Silesia in Katowice, Poland
}

\begin{abstract}
Objectives: Inflammation has been revealed to be associated with angiogenesis. Granulomatosis with polyangiitis (GPA) and immune complex small vessel vasculitis (ICSVV) are forms of systemic vasculitides of different pathogenesis. GPA is a necrotizing granulomatosis and ICSVV is associated with inflammation of postcapillary venules induced by deposits of immune complexes. The aim of the study was to determine serum levels of angiostatin and endostatin, natural angiogenesis inhibitors, in patients with GPA and ICSVV as well as healthy individuals.

Material and methods: Two groups of patients with GPA (20 patients) and ICSVV (20 patients) as well as 20 controls were investigated. All patients were investigated before initiation of immunosuppressive therapy or administration of corticosteroids. Angiostatin and endostatin levels were assayed with the ELISA method.

Results: Enhanced serum levels of angiostatin and endostatin were found in patients with GPA but not in those suffering from ICSVV. In patients with GPA increased levels of angiogenesis inhibitors correlated with the disease activity. A correlation between angiostatin and endostatin levels was observed in all groups of investigated individuals.

Conclusions: It is suggested that formation of necrotizing granulation is associated with profound activation of angiogenesis and an increase in serum levels of inhibitors is a phenomenon occurring during blood vessel formation in the granulation tissue. The obtained results confirm involvement of angiogenesis in pathogenesis of at least some forms of vasculitides and suggest the need for continuation of investigations in this field.
\end{abstract}

Key words: granulomatosis with polyangiitis, endostatin, angiostatin, immune complex small vessel vasculitis.

\section{Introduction}

The vasculitides are a heterogeneous group of disorders of unknown etiology characterized by inflammation located within the blood vessel wall. Specific causes of the diseases remain unknown and their classification is mostly based on the size of affected vessels and their location within the body. The last published classification and nomenclature of vasculitides includes, for the first time, some etiological aspects. The group of small vessel vasculitides consists of diseases associated with occurrence in the serum of patients of anti-neutrophil cytoplasmic antibodies (ANCAs) and other group of vasculitides that are believed to be associated with deposition of immune complexes within the vessel wall. Thus, the nomenclature of the diseases included two "etiological" subgroups of small vessel vasculitis, ANCA-associated small vessel vasculitis and immune complex small vessel 
vasculitis [1]. Detection of ANCA antibodies is possible due to the use of immunofluorescence (IF) on ethanol fixed neutrophils, and the use of formalin-fixed neutrophils makes it possible to identify types of antibodies, including the two most important, cytoplasmic (c-ANCA) and perinuclear ( $\mathrm{p}-\mathrm{ANCA}$ ).

Granulomatosis with polyangiitis (GPA) is a typical representative of the ANCA-associated small and medium-size vessel vasculitides. GPA is a relatively rare form of systemic vasculitis, characterized by necrotizing granulomatous involvement of the upper and lower airways and focal necrotizing glomerulonephritis. Necrotizing granulomatosis is a predominant pathological feature and is associated in most cases with a high serum level of c-ANCA antibodies. These antibodies have been suggested to take part in pathogenesis of the disease [2-6].

Immune complex small vessel vasculitis (ICSVV) is a more heterogeneous group of vasculitides. According to the proposed definition the group consists of "vasculitis with moderate to marked vessel wall deposits of immunoglobulins and/or complement components predominantly affecting small vessels" [1]. The most common diseases of the group are IgA vasculitis (formerly known as Henoch-Schönlein purpura) and cryoglobulinemic vasculitis. Cutaneous involvement (known as "palpable purpura"), common in patients with ICSVV, is related to the former name of the disease, leukocytoclastic vasculitis [6-8].

In vasculitis myointimal proliferation, fibrosis and thrombus formation leading to stenosis or occlusion of the vascular lumen occur, and it causes tissue ischemia. In the case of ischemia angiogenesis becomes a compensatory mechanism. Angiogenesis is a process of new vessel formation. Angiogenesis in contrast to vasculogenesis takes place, for the most part, after the fetal period and is involved in several physiological and pathological phenomena, including malignancy and a number of non-malignant disorders [9]. The mechanism of angiogenesis is very complex and is only partially elucidated. There are several suggestions that factors regulating inflammation also affect angiogenesis. For example, acute-phase serum amyloid A was found to be a regulatory factor of angiogenesis [10]. In recent years, a number of angiogenesis-stimulating and angio-

Table I. Characteristics of patients and controls

\begin{tabular}{|lccc|}
\hline & $\begin{array}{c}\text { Controls } \\
n=20\end{array}$ & $\begin{array}{c}\text { GPA } \\
n=20\end{array}$ & $\begin{array}{c}\text { ICSVV } \\
n=20\end{array}$ \\
\hline Age (years) & $39 \pm 5$ & $41 \pm 7$ & $36 \pm 8$ \\
\hline Gender (M/F) & $9 / 11$ & $9 / 11$ & $11 / 9$ \\
\hline Disease activity & - & $22.5 \pm 6.1$ & $20.0 \pm 5.2$ \\
\hline
\end{tabular}

GPA - granulomatosis with polyangiitis; ICSVV - immune complex small vessel vasculitis genesis-inhibiting factors have been reported. The bestknown natural inhibitors of angiogenesis are angiostatin and endostatin.

Angiostatin is a fragment of plasminogen with a molecular weight of about $38 \mathrm{kDa}$. A few forms of angiostatin express inhibiting activity. They are products of different proteolytic degradation of plasminogen. Angiostatin blocks a few stages of angiogenesis: proliferation, migration, differentiation of the endothelial cells and tube formation [11, 12]. Endostatin is a $20 \mathrm{kDa}$ fragment of collagen type XVIII, a component of the extracellular matrix of the connective tissue. It is a strong inhibitor of several stages of blood formation, although the detailed mechanism of its action is poorly understood [13-15].

The present study was designed to determine the serum level of natural angiogenesis inhibitors, angiostatin and endostatin in patients with two different forms of vasculitis. In general, the possibility of altered regulation of angiogenesis was evaluated in patients with GPA and ICSVV. These specific vasculitides are characterized by different pathological features, and different pathomechanisms are probably involved in development of vascular inflammation.

\section{Material and methods}

Twenty patients with the final diagnosis of GPA and 20 patients with ICSVV were investigated. All patients with GPA fulfilled classification criteria of the American Rheumatism Association [16]. All patients were c-ANCA positive. Patients with ICSVV were diagnosed on the basis of the clinical picture and in 14 cases with positive results of the microscopic evaluation of the skin biopsy. In the ICSVV group 17 patients suffered from IgA vasculitis and 3 from cryoglobulinemic vasculitis due to mixed cryoglobulinemia. The control group consisted of 10 healthy volunteers. The characteristics of the patients and controls are summarized in Table I. Activity of vasculitis was determined using the Birmingham Vasculitis Activity Score (BVAS) [17]. All patients were investigated before initiation of immunosuppressive therapy or administration of corticosteroids.

Venous blood samples were obtained in the morning after overnight fasting. Angiostatin and endostatin levels were assayed with the ELISA method using commercially available kits (Citimmune Sciences, College Park, MD, USA).

The study obtained a Bioethic Committee agreement.

\section{Results}

Table II summarizes results of angiostatin and endostatin measurements. A significant increase in angiostatin and endostatin levels was found in the GPA patients. 
Table II. Serum levels of angiostatin and endostatin

\begin{tabular}{|lccc|}
\hline Index & Controls & GPA & ICSVV \\
\hline Angiostatin $(\mathrm{ng} / \mathrm{ml})$ & $208.3 \pm 105.4$ & $1399.6 \pm 503.3$ & $427.5 \pm 187.0$ \\
\hline Endostatin $(\mathrm{ng} / \mathrm{ml})$ & $8.96 \pm 0.62$ & $25.11 \pm 1.15$ & $10.51 \pm 0.91$ \\
\hline
\end{tabular}

GPA - granulomatosis with polyangiitis; ICSVV - immune complex small vessel vasculitis

Statistical significance of differences: angiostatin: $A-B p<0.001, A-C N S, B-C p<0.001$; endostatin: $A-B p<0.001, A-C N S, B-C p<0.001$; NS - non-significant

Table III. Correlation of investigated indices

\begin{tabular}{|c|c|c|c|}
\hline Correlated indices & Controls & GPA & ICSVV \\
\hline Angiostatin/endostatin & $\begin{array}{l}r=0.45 \\
p<0.05\end{array}$ & $\begin{array}{l}r=0.38 \\
p<0.05\end{array}$ & $\begin{array}{l}r=0.31 \\
p<0.05\end{array}$ \\
\hline Angiostatin/disease activity & - & $\begin{array}{l}r=0.36 \\
p<0.05\end{array}$ & $\begin{array}{c}r=0.18 \\
N S\end{array}$ \\
\hline Endostatin/disease activity & - & $\begin{array}{l}r=0.37 \\
p<0.05\end{array}$ & $\begin{array}{c}r=0.11 \\
N S\end{array}$ \\
\hline
\end{tabular}

GPA - granulomatosis with polyangiitis; ICSVV - immune complex small vessel vasculitis; NS - non-significant

In contrast, the investigated angiogenesis inhibitors were only slightly enhanced in the ICSVV patients as compared with the controls. An increase in angiostatin and endostatin correlates with the disease activity in the GPA patients but in the ICSVV patients and controls there was no such correlation. A correlation between angiostatin and endostatin levels was observed in all groups of investigated individuals (Table III).

\section{Discussion}

The observation about the difference between GPA and ICSVV group is striking. This finding is concomitant with earlier suggestions of different pathogenesis of the investigated vasculitides. The most important pathological difference in development of necrotizing granulation in GPA patients compared to ICSVV is that this process is associated with new vessel formation and granulation tissue development and is characterized by growth of a dense vascular network. This is a cause of common bleedings in GPA patients, while in patients with ICSVV inflammation is mostly limited to the vascular wall and its surrounding.

Intense vessel formation in patients with GPA is believed to be associated with high activity of pro-angiogenic factors. In view of this suggestion, a high level of angiogenesis inhibitors can be considered as a homeostatic mechanism limiting activity of angiogenesis to granulation tissue. This hypothesis is supported by the correlation of serum levels of both inhibitors with the disease activity. It can be assumed that disease activity reflects, at least partially, the volume of newly formed granulation tissue. The above-described hypothesis needs further investigations.

It should also be mentioned that GPA is generally a more serious disorder than ICSVV [6]. GPA is usually associated with a higher level of inflammatory indices than ICSVV. This may be an additional explanation of the difference in angiogenesis inhibitor level between patients of the investigated groups.

Studies in other forms of vasculitis revealed that the diseases can be associated with altered angiogenesis. Keskin et al. [18] observed an increased level of angiostatin in patients with Behçet's disease and the elevation of serum angiostatin was associated with the disease activity. An imbalance between endostatin and an angiogenesis-stimulating factor (vascular endothelial growth factor) was found in patients with Kawasaki disease [19].

\section{Conclusions}

The obtained results confirm involvement of angiogenesis in pathogenesis of at least some forms of vasculitides and suggest the need for continuation of investigations in this field.

The study was conducted within the Research Project of the Medical University of Silesia, no. KNW-1-167/10.

The authors declare no conflict of interest.

\section{References}

1. Jennette JC, Falk RJ, Bacon PA, et al. 2012 Revised International Chapel Hill Consensus Conference Nomenclature of Vasculitides. Arthritis Rheum 2013; 65: 1-11. 
2. Lamprecht P, Kerstein A, Klapa S, et al. Pathogenetic and clinical aspects of anti-neutrophil cytoplasmic autoantibody-associated vasculitides. Front Immunol 2018; 9: 680.

3. Villa-Forte A. European League Against Rheumatism - European Vasculitis Study Group Recommendations for the Management of Vasculitis. Curr Opin Rheumatol 2010; 22: 49-53.

4. Sznajd J, Mukhtyar C. How to treat ANCA-associated vasculitis: practical messages from 2016 EULAR/ERA-EDTA recommendations. Pol Arch Med Wewn 2016; 126: 781-788.

5. Masiak A, Zdrojewski Z, Pęksa R, et al. The usefulness of histopathological examination of non-renal biopsies in the diagnosis of granulomatosis with polyangiitis. Reumatologia 2017; 55: 230-236.

6. Kucharz EJ. Vasculitides. In: Treatment of rheumatic disorders, Zimmermann-Górska I. (ed.). PZWL Wydawnictwo Lekarskie, Warszawa 2018: 204-251 (in Polish).

7. Claudy A. Pathogenesis of leukocytoclastic vasculitis. Eur J Dermatol 1998; 8: 75-79.

8. Kolopp-Sarda MN, Miossec P. Cryoglobulins: An update on detection, mechanisms and clinical contribution. Autoimmun Rev 2018; 17: 457-464

9. Kotulska A, Kucharz EJ. Angiogenesis in rheumatoid arthritis. Reumatologia 2011; 49: 1-9.

10. O'Neill L, Rooney P, Molloy D, et al. Regulation of inflammation and angiogenesis in giant cell arteritis by acute-phase serum amyloid A. Arthritis Rheum 2015; 67: 2447-2456.

11. Kotulska A, Mazurek U, Kotyla P, et al. Angiostatin, a natural inhibitor of angiogenesis. Por Farmaceut 2006; 1: 1-4 (in Polish).
12. Wahl ML, Kenan DJ, Gonzalez-Gronow M, et al. Angiostatin's molecular mechanism: aspects of specificity and regulation elucidated. J Cell Biochem 2005; 96: 242-261.

13. O'Reilly MS, Boehm T, Shing Y, et al. Endostatin: on endogenous inhibitor of angiogenesis and tumor growth. Cell 1997; 88: 277-285.

14. Kucharz EJ, Kotulska A. Pathophysiology of endostatin in non-malignant diseases. Pol Arch Med Wewn 2006; 115: $507-$ 511 (in Polish)

15. Walia A, Yang JF, Huang YH, et al. Endostatin's emerging roles in angiogenesis, lymphangiogenesis, disease, and clinical applications. Biochim Biophys Acta 2015; 1850: 2422-2438.

16. Leavit RY, Fauci AS, Bloch DA, et al. The American College of Rheumatology 1990 criteria for the classification of Wegener's granulomatosis. Arthritis Rheum 1990; 33: 1101-1107.

17. Stone JH, Hoffman GS, Merkel PA, et al. A disease-specific activity index for Wegener's granulomatosis. Arthritis Rheum 2001; 44: 912-920.

18. Keskin D, Keskin G, Inal A, et al. Serum angiostatin levels in patients with Behçet's disease: does angiogenesis play a role in the pathogenesis of Behçet's disease? Acta Clin Belg 2014; 69: 246-216.

19. Takeshita S, Kawamura Y, Takabayashi H, et al. Imbalance in the production between vascular endothelial growth factor and endostatin in Kawasaki disease. Clin Exper Immunol 2005; 139: 575-579. 